\title{
Electron Diffraction Based Analysis of Phase Fractions and Texture in Nanocrystalline Thin Films, Part III: Application Examples
}

\author{
J.L. Lábár ${ }^{1, \star}$ M. Adamik, ${ }^{1}$ B.P. Barna, ${ }^{1}$ Zs. Czigány, ${ }^{1}$ Zs. Fogarassy, ${ }^{1}$ Z.E. Horváth, ${ }^{1}$ O. Geszti, ${ }^{1}$ \\ F. Misják, ${ }^{1}$ J. Morgiel, ${ }^{2}$ G. Radnóczi, ${ }^{1}$ G. Sáfrán, ${ }^{1}$ L. Székely, ${ }^{1}$ and T. Szüts ${ }^{1,3}$ \\ ${ }^{1}$ Research Institute for Technical Physics and Materials Science, H-1121 Budapest, Konkoly-Thege M. út 29-33, Hungary \\ ${ }^{2}$ Institute of Metallurgy and Materials Science, Polish Academy of Sciences, 25 Reymonta st., 30-059 Kraków, Poland \\ ${ }^{3}$ Program in Biophysics, Harvard University, Cambridge, MA, USA
}

\begin{abstract}
In this series of articles, a method is presented that performs (semi)quantitative phase analysis for nanocrystalline transmission electron microscope samples from selected area electron diffraction (SAED) patterns. Volume fractions and degree of fiber texture are determined for the nanocrystalline components. The effect of the amorphous component is minimized by empirical background interpolation. First, the twodimensional SAED pattern is converted into a one-dimensional distribution similar to X-ray diffraction. Volume fractions of the nanocrystalline components are determined by fitting the spectral components, calculated for the previously identified phases with a priori known structures. These Markers are calculated not only for kinematic conditions, but the Blackwell correction is also applied to take into account dynamic effects for medium thicknesses. Peak shapes and experimental parameters (camera length, etc.) are refined during the fitting iterations. Parameter space is explored with the help of the Downhill-SIMPLEX. The method is implemented in a computer program that runs under the Windows operating system. Part I presented the principles, while part II elaborated current implementation. The present part III demonstrates the usage and efficiency of the computer program by numerous examples. The suggested experimental protocol should be of benefit in experiments aimed at phase analysis using electron diffraction methods.
\end{abstract}

Key words: electron diffraction, SAED, ring patterns, nanocrystals, thin films, phase fractions, texture, quantitative analysis, TEM, computer program

\section{INTRODUCTION}

Our method determines the quantity of known phases from the measured diffraction pattern, but does not attempt to determine the structure of any unknown phases. To understand in detail how the results in the present article are deduced from the measured selected area electron diffraction (SAED) patterns in the transmission electron microscope (TEM), the reader is referred to the first two parts of this series of articles that elaborated principles (Lábár, 2008) and implementation (Lábár, 2009) of the method. A brief summary of the procedure follows. On the basis of preliminary information (e.g., composition, etc.), phases in a list of known crystalline phases of the given element combinations are examined one-by-one if their diffracted lines are present in the measured diffraction pattern. Judgment is based on visual comparison of the measured peaks to calculated Marker lines that show both the positions and intensities of the diffracted lines of the known phase. The result of this qualitative phase identification is a shortlist of phases that will be attempted to fit in the next quantitative step. ${ }^{a}$ The diffracted lines of the phases in the short list (the

\footnotetext{
${ }^{a}$ Although information about the structure of known phases can be obtained from the XRD database, it does not mean by any means that we would need XRD prior to applying our method. Qualitative identification of the phases is also done with SAED.

Received March 26, 2011; accepted October 25, 2011

*Corresponding author. E-mail: labar@mfa.kfki.hu
}

"Model") must explain all significant peaks in the measured distribution, and all significant peaks of the Model must also be present in the measured distribution.

$\mathrm{Up}$ to that point the procedure is identical to that followed with X-ray diffraction (XRD) powder patterns prior to Rietveld phase analysis. During the calculation of the mentioned kinematic diffracted intensities, the scattering amplitudes of Weickenmeier and Kohl (1991) are used. Whenever the positions of the measured diffracted lines agree with the Model but the relative intensities significantly deviate (lines are missing in the extreme case), it is examined if the systematic deviation can be explained by the presence of fiber texture. In the present implementation only extremely sharp fiber texture is assumed, and no angular variance around the texture axis is allowed for. Partially textured phases are treated as a mixture of extremely sharp textured grains and another volume of randomly oriented ones. Their volume fractions are determined as separate quantities.

If the above procedure results in an unambiguous Model, we fit this model as described below. If several Model variants are conceived, they must be fitted one-byone, and the most probable solution is selected on the basis of the quality of the fit.

The fitting procedure starts with a construction of model functions in a parametric form to describe both the background and all the peaks. The shape of the background 
is determined empirically, putting a smooth curve under the peaks. The reader is reminded that the background in $\mathrm{XRD}$ is also determined empirically. For the peak shapes our method uses the pseudo-Voigt function, which is a linear combination of a Gaussian and a Lorentzian. Both the (generally common) widths of the Gaussian and the Lorentzian and their intensity ratio are treated as fitting parameters. In the present implementation the peak widths are empirical parameters, and no attempt was made to correlate them with grain size. The parameter space is explored by the SIMPLEX method to find the set of parameters that result in minimal deviation between measured and modeled distributions. The relative intensities, characteristic of the given phase, are kept constant during the fitting procedure, so the best fit gives an estimate for the volume fractions of the phases (and for their textured fractions). The most significant differences from the Rietveld method are the scattering amplitudes and the parameter optimization method. ${ }^{\mathrm{b}}$

Although certain parts of the experimental setup described below might seem self-evident, the authors recommend specific procedures that might be overlooked by the casual TEM user. The suggested experimental protocol ensures reproducible calibration of the TEM camera length with $0.3 \%$ reliability, which is an order of magnitude better than that experienced by the casual user.

The body of this article starts with suggestions on how to record a diffraction pattern optimal for quantification, followed by a description of the preparation of samples used in our examples. Examples of pattern processing and their results start with simple issues such as peak shapes and extending the dynamic range, followed by examining the precision of calibration that determines how reliably lattice parameters can be measured. Examples for the analysis of phases and texture progress from simpler ones to the more complicated case of seriously overlapping diffraction peaks, and a method is given on how to overcome the limitation of overlap for some of those problematic samples. The article concludes by showing the advantages of SAED over XRD and estimating detection limits, accuracy, and precision.

\section{Methods and Materials}

\section{Experimental Parameters to Optimize during Recording SAED Patterns}

The presence of texture must always be checked by tilting the sample and observing if the intensity distribution along the rings changes or not. If texture is present, the sample must be tilted so that the texture axis coincides with the electron beam, ensuring uniform intensity distribution along the rings.

In contrast to the XRD experiments, lenses are present in the TEM with both variable focal lengths and unavoid-

\footnotetext{
${ }^{\mathrm{b}}$ The SIMPLEX is semiglobal in contrast to the local nature of the Rietveld optimization.
}

able distortions. Furthermore, many microscopists optimize the settings of lenses to enhance visibility of their images and disregard the quality and reproducibility of the SAED patterns (camera length, line widths, etc.). As a consequence, there is a general judgment that the reproducibility of the recorded pattern's camera length is not better than 1-3\% (Williams \& Carter, 1996). Since most people do not evaluate the ring width of a SAED pattern, they are not concerned with the exact value of beam convergence. Although it is a known fact that ring diameter may differ slightly in different directions on the SAED pattern, these distortions are not really corrected for during manual evaluation. For a reproducible and quantifiable experiment, all of the above issues must be addressed.

Since the current of the objective lens is generally kept constant by most microscopists, the two most important experimental parameters that influence the camera length are the position (in $z$ direction) of the sample and the currents of the condenser lenses (whose fields interact with each other and with that of the objective lens). If not only the objective current but also the condenser currents are controlled carefully, the camera length will be reproduced within $0.3 \%$ with the following protocol (which means an order of magnitude improvement). After each sample change, all lenses must be demagnetized ("Normalization" function in case of Philips microscopes), and the current of the objective lens must be set to the prespecified value. The next step of the procedure we recommend is that the height of the sample must be set to the correct value. Then, after selecting the area of interest, the current of the last condenser lens (generally C2) must be set to the prespecified value and the sharpness of the rings must be checked by focusing the projection system. If the first two are correctly set, no focusing adjustment should be needed for the projecting system (once this value has been properly determined). Based on these principles the following procedure is recommended to record SAED patterns. Sample height can be set while the image is observed at a magnification of 10,000 or similar. By focusing the $\mathrm{C} 2$ lens in imaging mode [objective aperture (OA) and selected area aperture (SAA) are removed], any of the three image types in Figure 1 are seen. By adjusting the sample height mechanically, the spot with minimum diameter in Figure $1 \mathrm{~b}$ should be reached. Open the illumination by turning the $\mathrm{C} 2$ knob clockwise until the beam is almost parallel [the size of the $\mathrm{C} 2$ aperture (C2A) and the currents of the condenser lenses (spot size and IC2) determine the value of beam convergence]. When a "standard" (i.e., known crystalline powder) is recorded with these values and the camera length is calibrated for its SAED pattern, the reproducibility of the camera length will be within $0.3 \%$, and we adhere to the above procedure and keep the same values while recording an SAED pattern from an unknown sample. For reproducible camera length, a wide range of IC2 can be preselected; however, the width of the rings is also affected by that value. The more parallel the beam (the smaller the convergence angle), the sharper the rings. Ring width is a slowly varying function for a broad 


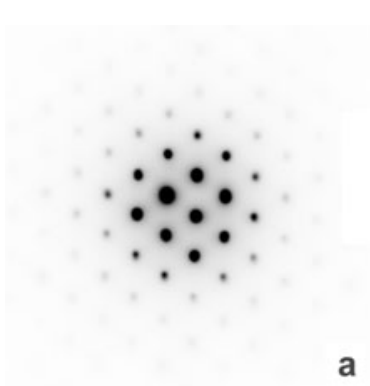

b

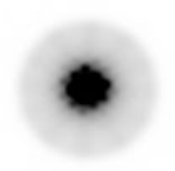

C

Figure 1. Inverted BF image of a focused beam in TEM. a: The sample is above the nominal object plane (a small diffraction pattern is seen in image mode). b: The sample is at the object plane of the objective lens (a sharp point is seen in image mode). c: The sample is below the object plane (a "skirt" around the point is seen in image mode).

range of IC2, with sharp increase as the focusing condition of $\mathrm{C} 2$ is approached (see Fig. 2a). The variation of the camera length as a function of IC2 in the same range is almost linear (Fig. 2b). Since intensity is also reduced by opening the illumination (reducing convergence), a good trade-off must be found. Refining the camera length better than $0.3 \%$ can be done with the fitting procedure that is elaborated in the present article. Significant alteration in the stigmator settings also influences the measured value of the camera length, so recalibration is also suggested after major adjustments of the stigmator. Lens distortions also determine the "ellipticity" of the measured SAED patterns, so this noncircular distortion of the pattern is also kept constant by selecting the fixed settings.

Due to the spherical aberration of the objective lens, the width of the diffraction rings is also a function of the size of the sample area selected for diffraction. Figure 3 shows the peak width measured as a function of the size of the SAA while all other parameters are kept constant (the change in intensity is balanced by an appropriate change in exposure time). The new generation of $C_{s}$-corrected TEMs should bring a serious improvement in that field, too.

Elliptical distortion is corrected for by a procedure that is elaborated in Part II (Lábár, 2009, p. 22). Incomplete correction of the distortion also spoils peak shape and the quality of the fit and also affects the measured value of the camera length.

Stray radiation, whose presence is not even recognized by many microscopists, has to be considered. If the illumination covers a thick part of the sample or the grid itself, it is a source of stray radiation even if the area of interest is
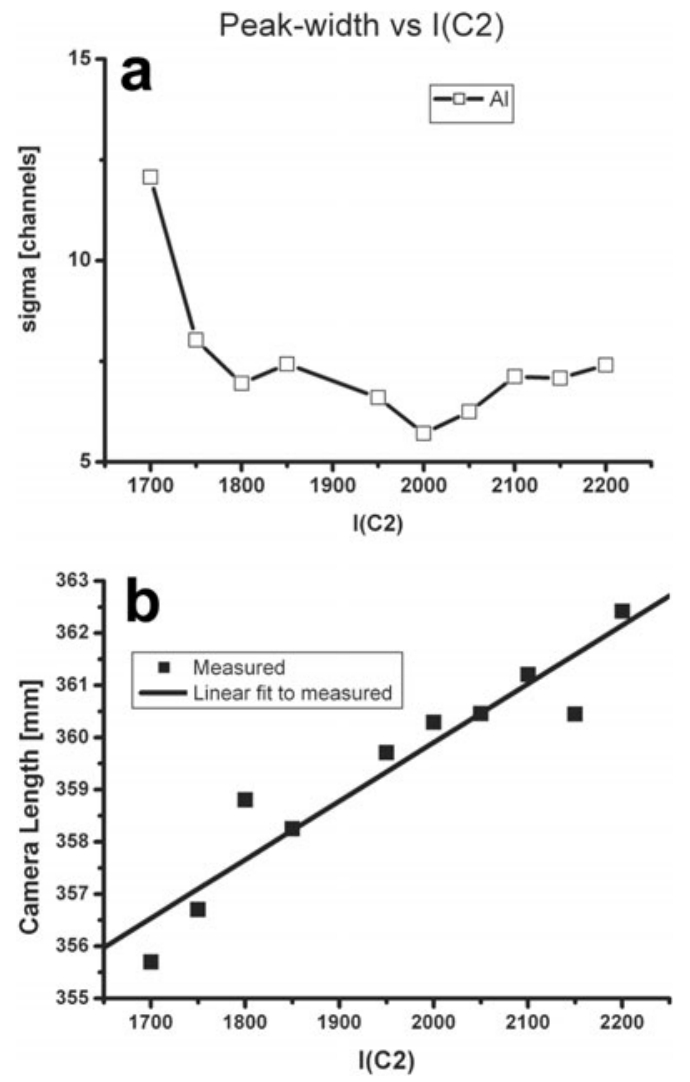

Figure 2. a: Dependence of the measured peak width on the current of the second condenser lens, while all other parameters were held constant. (Taken from the Al-Ge sample in Fig. 6.) b: Dependence of the camera length on the current of the second condenser lens, while all other parameters were kept unaltered.

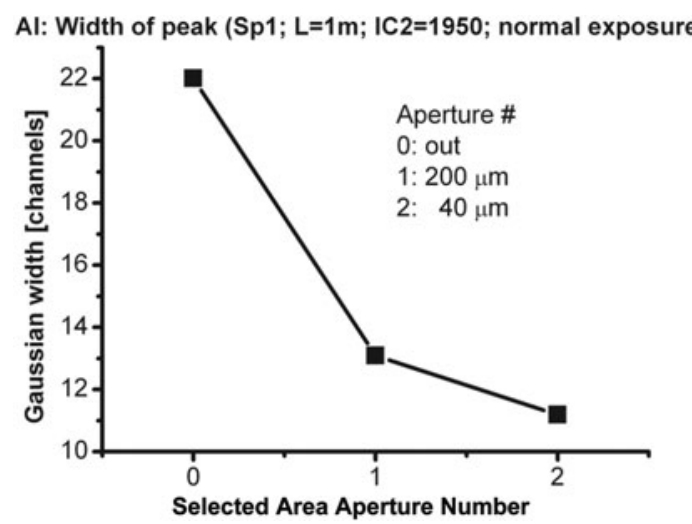

Figure 3. Dependence of the measured peak width on the size of the SAA, while all other parameters were kept unaltered.

localized by the SAA. In many cases it results in an increased background under the peaks, with background intensity increasing toward higher scattering angles-just the reverse of normal behavior. The uneven background is best seen on a logarithmic intensity scale (Fig. 4a). Circular (elliptical) averaging does not eliminate this problem. The effect is best seen in the one-dimensional (1D) distribution (Fig. 4b). Careful reduction of the illuminated area (in contrast to only restricting the area of interest by the SAA) eliminates, 

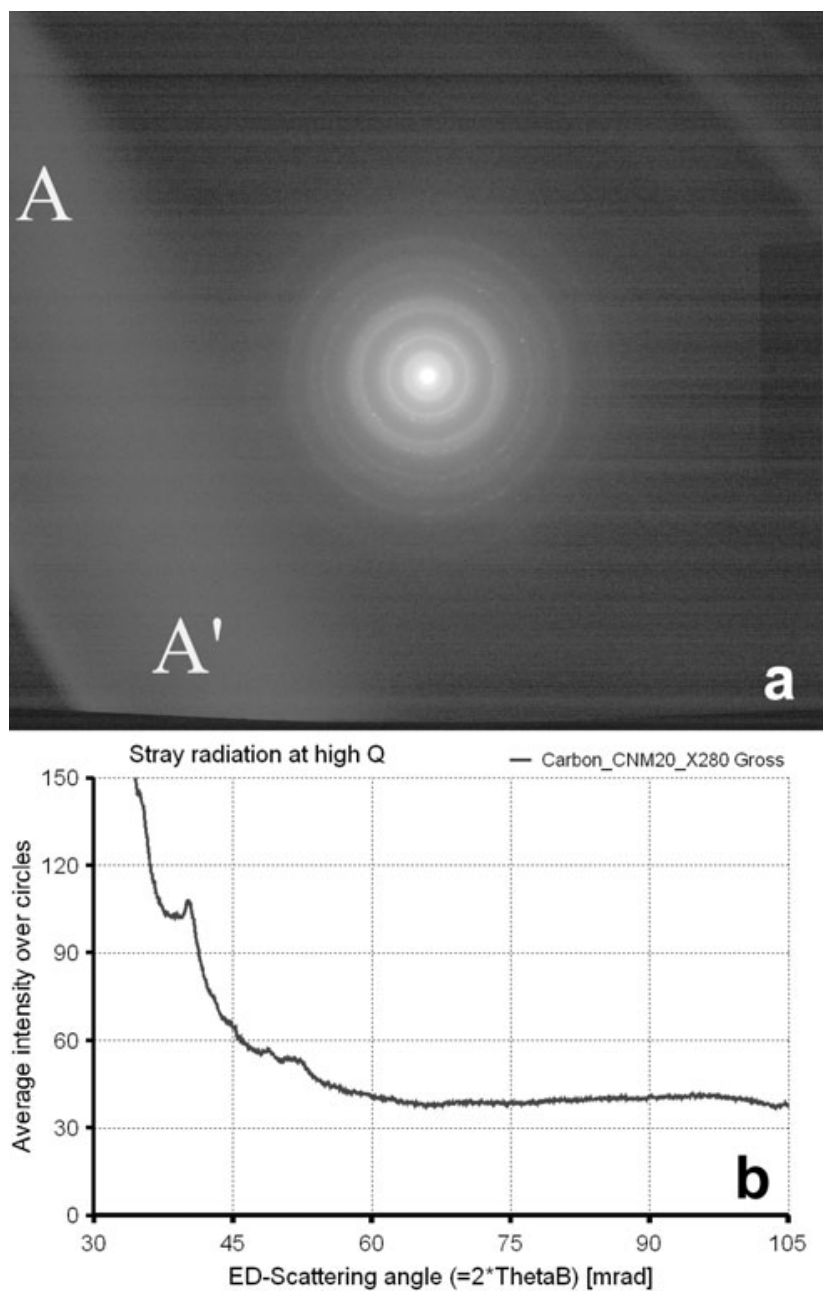

Figure 4. The effect of stray radiation scattered from a nearby grid bar. a: The original 2D pattern. The intensity is in logarithmic scale to ensure better visibility over a wide intensity range. An SAA of $40 \mu \mathrm{m}$ diameter was applied. The region along letters $\mathrm{A}-\mathrm{A}^{\prime}$ shows the anomaly due to the stray electrons scattered from a nearby grid. SAA could not eliminate this problem completely. b: Circularly averaged intensity distribution. An increase is observed at the high angle range of the $1 \mathrm{D}$ distribution due to the stray radiation, in contrast to an expected decrease, which should be observed in a normal case. The anisotropy of the original distribution is smeared off by the averaging over all angles.

or at least alleviates, that problem. Changed IC2 to reduce the illumination may also require recalibrating of the camera length as well. If not eliminated experimentally, that effect will spoil the quality of the background fit. However, if the background is tightly followed by a flexible function (such as the spline), the net peak intensities are less affected by the stray scattering.

The number of grains in the analyzed area will determine how continuous or spotty the rings will be. Grain size, together with the size of the area analyzed and sample thickness, determines the number of grains, i.e., statistics. If the number of grains is too low (the rings are spotty), perfect statistical averaging across directions cannot be expected. Consequently, the relative intensities of the rings might be slightly compromised by statistical fluctuations that cannot be corrected for by any computer programs.

\section{Characteristics and Preparation of Samples Suitable for Testing the Present Method}

TEM specimens of two components optimized for the analysis of phase fractions have to be small grained and thin to avoid coherent dynamic and incoherent double scattering. Pairs of components not forming compound phases at the given experimental conditions have to be selected. Besides, to eliminate background scattering of the support, they should be self-supporting or at least deposited on an amorphous support film as thin as possible.

For a comprehensive test of the method, numerous thin test layers of various elements and pairs of elements were prepared by physical and chemical methods. Self-supporting test layers were deposited onto $\mathrm{NaCl}$ substrates, floated off in water, and placed on microgrids. Supports of the layers, if applied, were amorphous-Carbon (a-C) films of thickness up to a maximum of $15 \mathrm{~nm}$ (though mostly 5-10 nm). The thickness of the test films was limited to a maximum of $20 \mathrm{~nm}$. The components of the films were deposited either simultaneously or subsequently or as a stratified multilayer. Stratification was applied to limit the grain size of the films. For the same purpose, the substrate was unheated in most cases.

Reference layers of single elements, $\mathrm{Au}$ and $\mathrm{Pt}$, were deposited both by thermal evaporation in high vacuum (typically 2-8 $\times 10^{-5} \mathrm{mbar}$ ) and drip and dry technique from $\mathrm{Pt}$ sol, respectively. A group of element couples, $\mathrm{Cu} /$ $\mathrm{Ag}, \mathrm{Cr} / \mathrm{Ag}, \mathrm{Ni} / \mathrm{Ag}$, and $\mathrm{Al} / \mathrm{Ge}$, were deposited subsequently by thermal evaporation.

$\mathrm{Cr}_{x} \mathrm{Al}_{1-x} \mathrm{~N}$ samples were prepared by simultaneous DC magnetron sputtering of $\mathrm{Cr}$ and $\mathrm{Al}$ in nitrogen atmosphere in a stainless steel vacuum chamber of $5 \times 10^{-8} \mathrm{mbar}$ background pressure. The thickness of the components was measured by oscillating quartz crystal thickness monitors during deposition.

The composition of the test films was measured by a NORAN energy dispersive X-ray spectrometer (EDS) equipped with a $\mathrm{Ge}$ detector. Unintentionally oxidized samples were also identified and disregarded with the help of EDS analysis. The elemental compositions obtained by the EDS were used as reference. This procedure is based on the assumption that all of the evaporated elements are present in crystalline form, which means that elemental concentrations (measured by EDS) are equivalent to crystalline volume fractions (determined by diffraction).

SAED patterns measured in a Philips CM-20 TEM were recorded on imaging plates (ensuring 20 bit dynamic range) and were evaluated by the ProcessDiffraction procedure (http://www.mfa.kfki.hu/ labar/ProcDif.htm).

\section{Results}

\section{Peak Shapes}

Gaussian, Lorentzian, and Pseudo-Voigt shapes can be selected in the present implementation to approximate the 


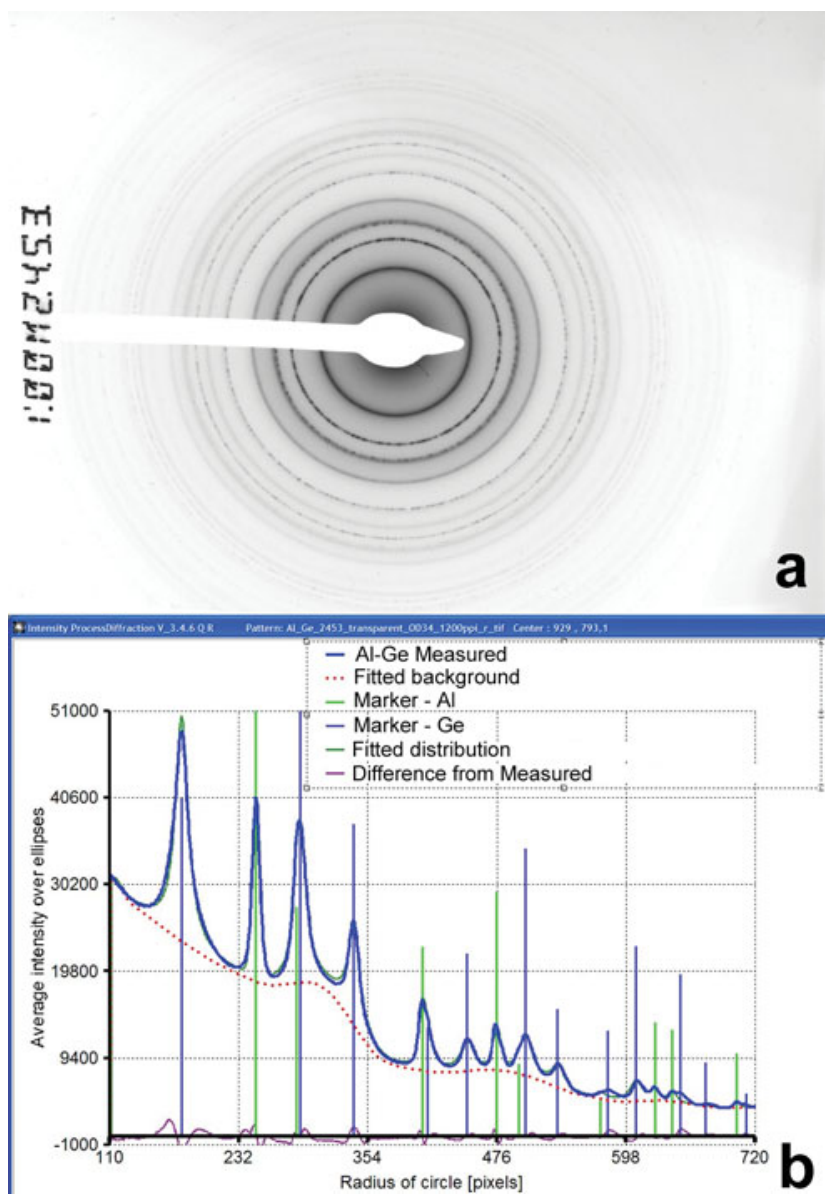

Figure 5. Different peak shapes and peak widths, characteristic of the two distinct components, within the same sample. a: The measured SAED pattern from a Ge-Al sample. b: The $1 \mathrm{D}$ distribution calculated from panel a, with markers of $\mathrm{Al}$ and $\mathrm{Ge}$. The different peak shapes and peak widths characteristic of the $\mathrm{Al}$ and the $\mathrm{Ge}$ nanocrystalline fractions are obvious.

shapes of peaks in the measured 1D distribution. An example that demonstrates the importance of selecting different peak shapes is given in Figure 5. The TEM test sample was prepared on a thin a-C supporting layer by sequential evaporation of $10 \mathrm{~nm} \mathrm{Ge}$ and $10 \mathrm{~nm} \mathrm{Al}$, as controlled by vibrating quartz thickness monitor. The Pseudo-Voigt peak shape was selected at the start for both components, but their shape parameters were allowed to be varied independently for the two components during the fitting procedure. The resulting peak shape characteristics of the $\mathrm{Al}$ phase are mainly Gaussian in shape and narrow, while the peak shape of the Ge nanocrystalline phase within the same sample is close to pure Lorentzian with increased width, as can be seen from Figure 5b. This example illustrates why it is important to independently optimize the peak shapes of each component phase in a single sample. Intermediate, Pseudo-Voigt peak shapes, with separately tunable parameters, are seen in all of the other examples in this article.

\section{Extending Dynamic Range by Merging}

Limitations of dynamic range of the recording medium are extremely conspicuous in the case of photographic films, where the dynamic range is at most 8-bits (256 gray levels). The linear range might even be more limited, depending on the type of film and the method and parameters of development. However, even the 16-bit (65,536 gray levels) dynamic range of most of the good slow charge-coupled device cameras or of one type of imaging plates (IP) is insufficient to record the entire range of intensities that might be present within a single diffraction pattern. A solution to the problem is offered by recording and merging an exposure series. Since subsequent pieces of either the film or the IP can be displaced relative to each other, first, the members of the series must be centered individually (Lábár, 2009). Merging is done for the $1 \mathrm{D}$ distributions that were deduced from the measured two-dimensional (2D) patterns. For a given pair of distributions, a section is identified where both distributions have intensity values within the linear range of the recording medium and a normalizing factor is determined to correct for the different exposures. Low intensity values are retained from the higher exposure, while intensity values of overexposure are replaced by the normalized value, taken from the lower exposure.

As an example, distributions from low and high exposures recorded from polycrystalline $\mathrm{Cu}$ are shown in Figures $6 \mathrm{a}$ and $6 \mathrm{~b}$. Oversaturated values are clearly seen in Figure $6 \mathrm{~b}$. Figure $6 \mathrm{c}$ shows the merged distribution. Correction of the overexposed parts and extension of the dynamic range are obvious. The normalized values in Figure $6 \mathrm{c}$ come from the high intensity distribution in Figure $6 \mathrm{~b}$ whenever the values are below $90 \%$ of the saturation value of that distribution $(230 \cong 0.9 \times 255$ in the example). Whenever the value in Figure $6 \mathrm{~b}$ is above this threshold, values from the low intensity distribution (Fig. 6a) are corrected for the differences in the illumination conditions between the two distributions. That latter correction factor is an automatically determined average ratio for channels, whose value is between $80 \%$ and $90 \%$ of the saturation value for Figure $6 b$.

\section{Precision of Calibration: Absolute Measurement of Lattice Parameter}

To test the reproducibility of setting the same camera length by the procedure elaborated above, a polycrystalline thin film of $\mathrm{Al}(20 \mathrm{~nm}$ thick, with lateral grain size of $40 \mathrm{~nm})$ was measured repeatedly. After each measurement, the sample was taken out of the TEM, the lens currents were intentionally altered, then the sample was reinserted into the TEM and the experimental resetting of parameters was repeated. The procedure was also repeated on consecutive days. Results of five such measurements were statistically processed. The average camera length was $941.2 \mathrm{~mm} \pm 2.49 \mathrm{~mm}$, while the minimum and maximum values were 938.6 and $943.5 \mathrm{~mm}$, respectively. On that basis we claim that the precision of our calibration is better than $0.3 \%$. Accuracy of the measured camera length can be further improved when 


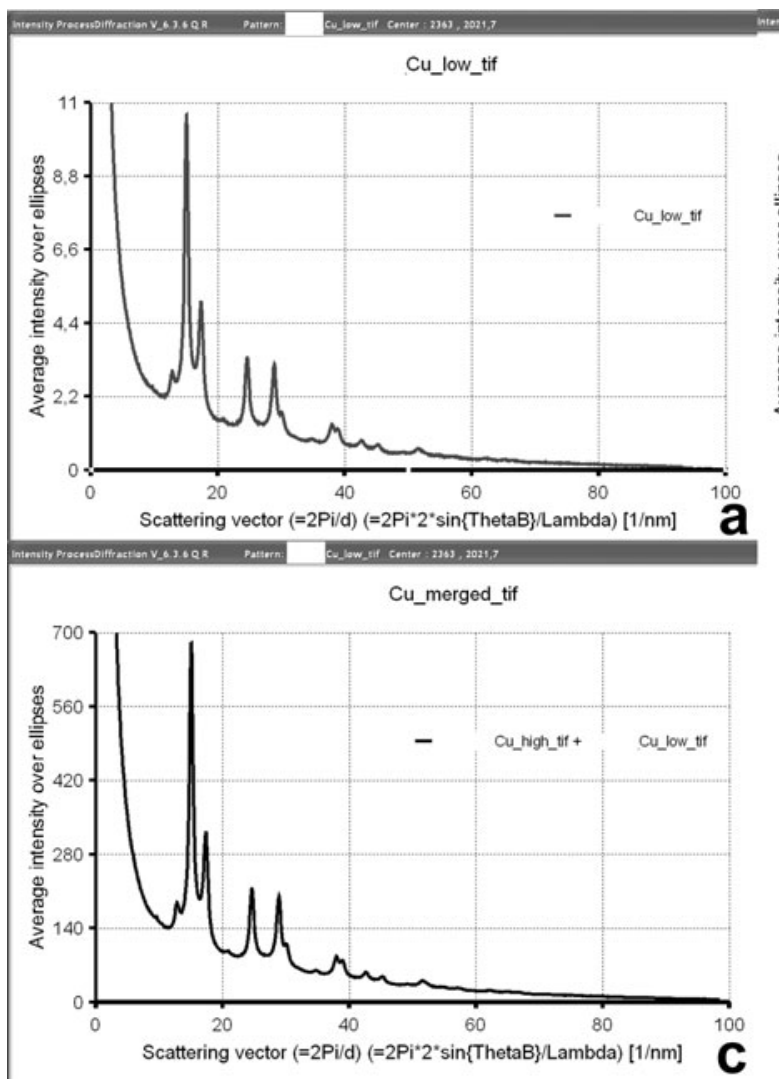

an "internal standard" exists, i.e., if a well-separated ring of a known phase can be identified in the diffraction pattern. In such cases $0.1 \%$ accuracy in the absolute camera length can be easily reached, and variation of the cell parameter of another phase within the sample can be measured with that improved accuracy.

If the change in the lattice parameter of a phase significantly exceeds this limit (e.g., due to alloying), the absolute change in the lattice parameter can be measured. Obviously, it is not as accurate and precise as the changes measured with XRD, but it gives useful semiquantitative information.

As an example, the change in the lattice parameter in $\mathrm{CrN}$ is measured. The change is induced by the addition of AlN, which was dissolved and formed a single phase facecentered cubic (fcc) $\mathrm{Cr}_{x} \mathrm{Al}_{1-x} \mathrm{~N}$ with altered lattice parameter. Polycrystalline Au was used for the calibration of the camera length. Generally we select polycrystalline material with grain size $>20 \mathrm{~nm}^{\mathrm{c}}$ to avoid miscalibration due to the change in lattice parameter of nanocrystalline material as compared to the bulk value (Goldstein et al., 1992). After calibration with $\mathrm{Au}$, the sample $\mathrm{Cr}_{x} \mathrm{Al}_{1-x} \mathrm{~N}$ was measured under identical conditions and the diffraction pattern was processed quantitatively, using a single phase $(\mathrm{CrN})$ for fitting. The camera constant was kept constant at the previously calibrated value, and the lattice parameter of $\mathrm{CrN}$ was used as a parameter to refine. Figure $7 \mathrm{c}$ shows the quality of

${ }^{c}$ During this calibration of the camera length, only the position of one well-separated line is determined for the reference material.

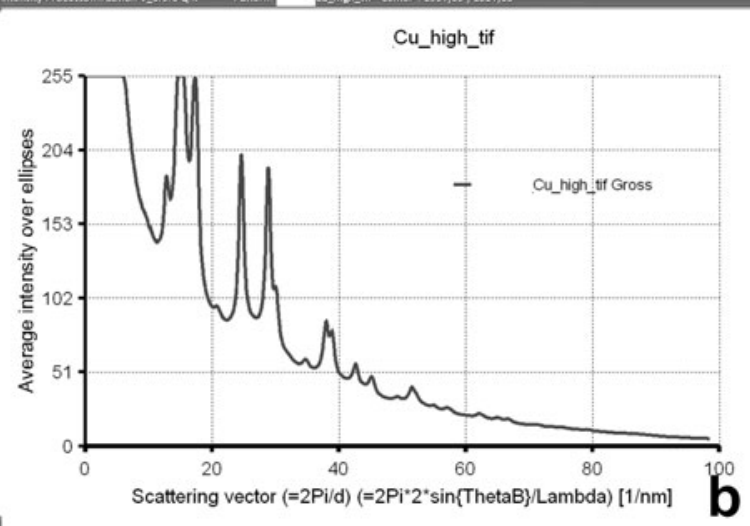

Figure 6. Intensity distribution deduced from the high exposure SAED pattern (a) and from the low exposure (b), together with the merged distribution (c).

the fit. The experimentally determined lattice parameter was $0.407 \mathrm{~nm}$, while the nominal value is $0.414 \mathrm{~nm}$, corresponding to $1.6 \%$ change. As compared to the standard deviation of the calibration $(0.3 \%)$, the change is accepted as significant. The example also shows the limitations of our approach: the absolute change in the lattice parameter can be determined only for relatively large changes. Smaller change can only be measured reliably when an internal standard is present in the sample.

Another example is given in Figure $7 \mathrm{~d}$ and Table 1. Three samples with different compositions were prepared by co-evaporation of $\mathrm{Cu}$ and $\mathrm{Mn}$. Their compositions were measured by EDS and the lattice parameters were determined by XRD. Cross sections from the same samples were also evaluated by SAED, using the ProcessDiffraction method. The results in Table 1 show that there is a good agreement between the lattice parameters measured by the two diffraction methods, supporting our claim for the precision of our

Table 1. Change in Lattice Parameter due to Dissolution of Mn in $\mathrm{Cu}$.

\begin{tabular}{lccc}
\hline & EDS & $\begin{array}{c}\text { Lattice } \\
\text { Parameter } a \\
(\AA) \text { by XRD }\end{array}$ & $\begin{array}{c}\text { Lattice } \\
\text { Parameter } a \\
(\AA) \text { by SAED } \\
( \pm 1 \sigma)\end{array}$ \\
\hline Cumple & $($ at.\%) $( \pm 1 \sigma)$ & 3.638 & $3.631 \pm 0.011$ \\
CuMn08 & $6 \pm 2$ & 3.675 & $3.651 \pm 0.011$ \\
CuMn02 & $31 \pm 2.5$ & 3.710 & $3.700 \pm 0.011$ \\
\hline
\end{tabular}



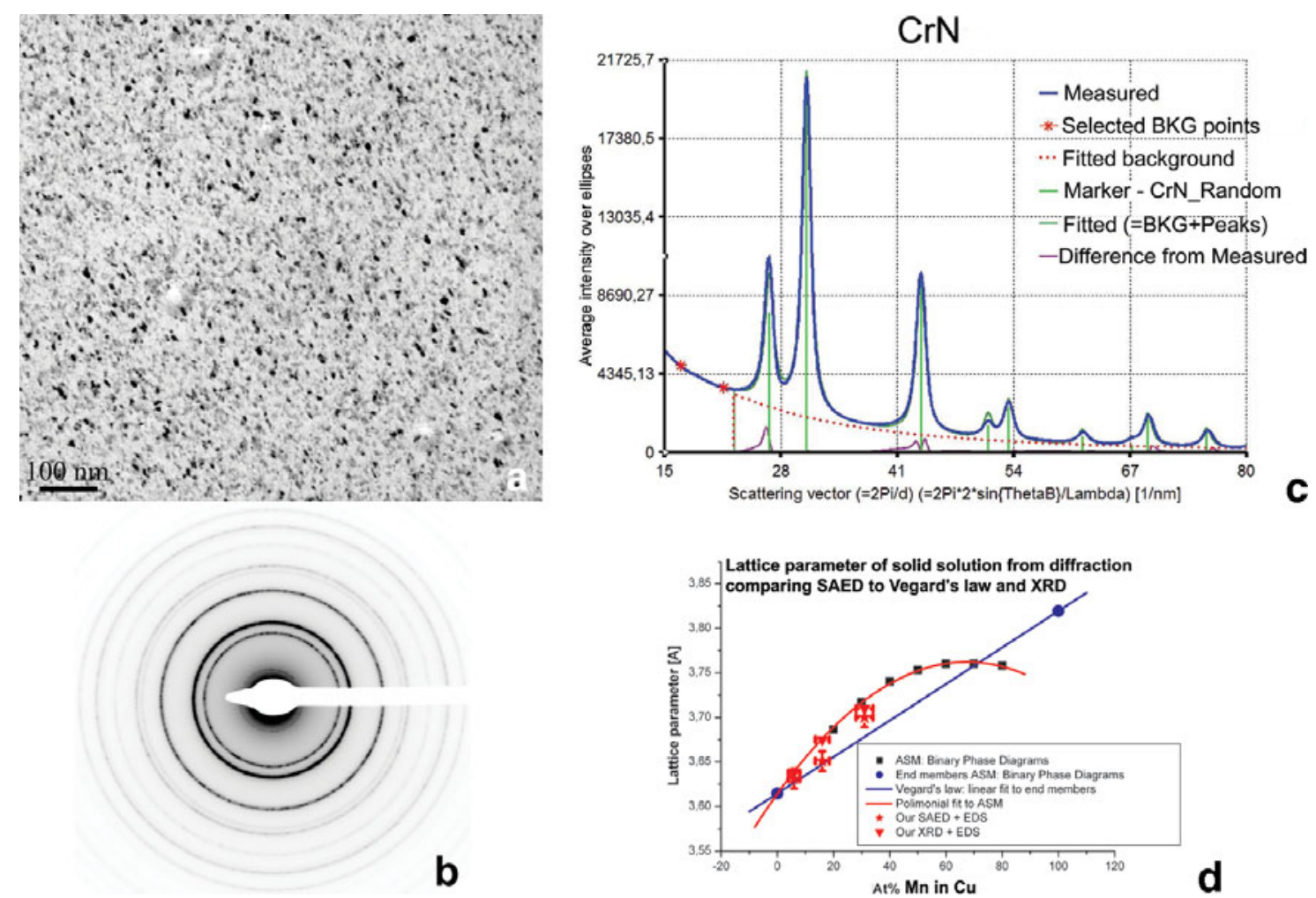

Figure 7. Absolute measurement of lattice parameter based on calibration of the camera length. Change in lattice parameter of fcc-CrN is induced by dissolved $\mathrm{Al}$ from co-sputtering of $\mathrm{Cr}$ and $\mathrm{Al}$ in nitrogen environment. EDS analysis shows that the metallic components are dominated by 70 at.\% $\mathrm{Al}$, while $\mathrm{Cr}$ is only 30 at.\%. We consider this structure that of $\mathrm{CrN}$ because it retained the fcc structure of $\mathrm{CrN}$, while AlN is hexagonal. Due to the different structures of $\mathrm{CrN}$ and AlN, the Vegard law cannot be applied to estimate the change in lattice parameter due to the dissolved Al. a: BF image, showing grain size of $10 \mathrm{~nm}$ in a thin layer with 20-30 nm sample thickness. b: Measured SAED pattern. c: Processed distribution, with fitted model function. The experimentally determined lattice parameter was $0.407 \mathrm{~nm}$, while the nominal value is $0.414 \mathrm{~nm}$, corresponding to $1.6 \%$ change. Compared to the standard deviation of the calibration $(0.3 \%)$, the change is significant. d: Lattice parameter of $\mathrm{Cu}(\mathrm{Mn})$ solid solution as a function of $\mathrm{Mn}$ concentration. Squares: Data cited in Binary Phase Diagrams with polynomial fit. Circles: Data cited in Binary Phase Diagrams from the end members with a linear fit that indicates Vegard's law. Deviation from Vegard's law is obvious. Triangles: Lattice parameter was measured by XRD and concentration was measured by EDS from same samples that were co-evaporated. Asterisk: Lattice parameter was measured by SAED and ProcessDiffraction and concentration was measured by EDS. Lattice parameters determined by SAED are more accurate than an estimate from Vegard's law.

lattice parameter measurements. Figure 7d shows data from the ASM Binary Phase Diagrams, with a polynomial fit to the data and with a linear fit to the end members, $\mathrm{Cu}$ and Mn, corresponding to Vegard's law. The ASM data indicate that Vegard's law is of limited accuracy. The triangles show our combined XRD and EDS measurements on the three samples and perfectly match previously cited values. Our SAED measurements are shown with the asterisk symbol: the composition from EDS is combined with the lattice parameter from SAED, as evaluated by ProcessDiffraction. Our result is better than the prediction by Vegard's law, but not as accurate as that obtained by XRD.

\section{Relative Measurement of Lattice Parameter}

For two-phase samples, the relative change in the lattice parameters of each phase can sometimes be determined even without accurate absolute calibration. In the case of such relative changes, the strong peak of one of the phases (as the one labeled "A" in Fig. 8c) can be used as internal standard, and the absolute calibration is not required to detect the relative change. The calibration can be carried out with that strong peak. In the next step, the camera length is kept at the calibrated value and the lattice constant of the second phase is refined. Obviously, it is the relative change that is measured here and the absolute values might be different if the lattice parameter of the first phase is also changed (which is likely). In this example, $\mathrm{Cu}$ and $\mathrm{Ag}$ are co-evaporated. The local composition and grain structure are determined by EDS and bright-field (BF)/dark-field (DF) TEM (Fig. 8a), respectively. Tilted samples show the presence of texture. The EDS composition was equivalent to 33 vol\% Ag and 67 vol\% Cu. Randomly-oriented and $\langle 111\rangle$ textured components were calculated for both phases (Fig. 8c). Our method shows $36 \mathrm{vol} \% \mathrm{Ag}$ and $64 \mathrm{vol} \% \mathrm{Cu}$, and most of both phases are $\langle 111\rangle$ textured (31 vol\% Ag and $51 \mathrm{vol} \% \mathrm{Cu}$ are in textured form), in agreement with results 

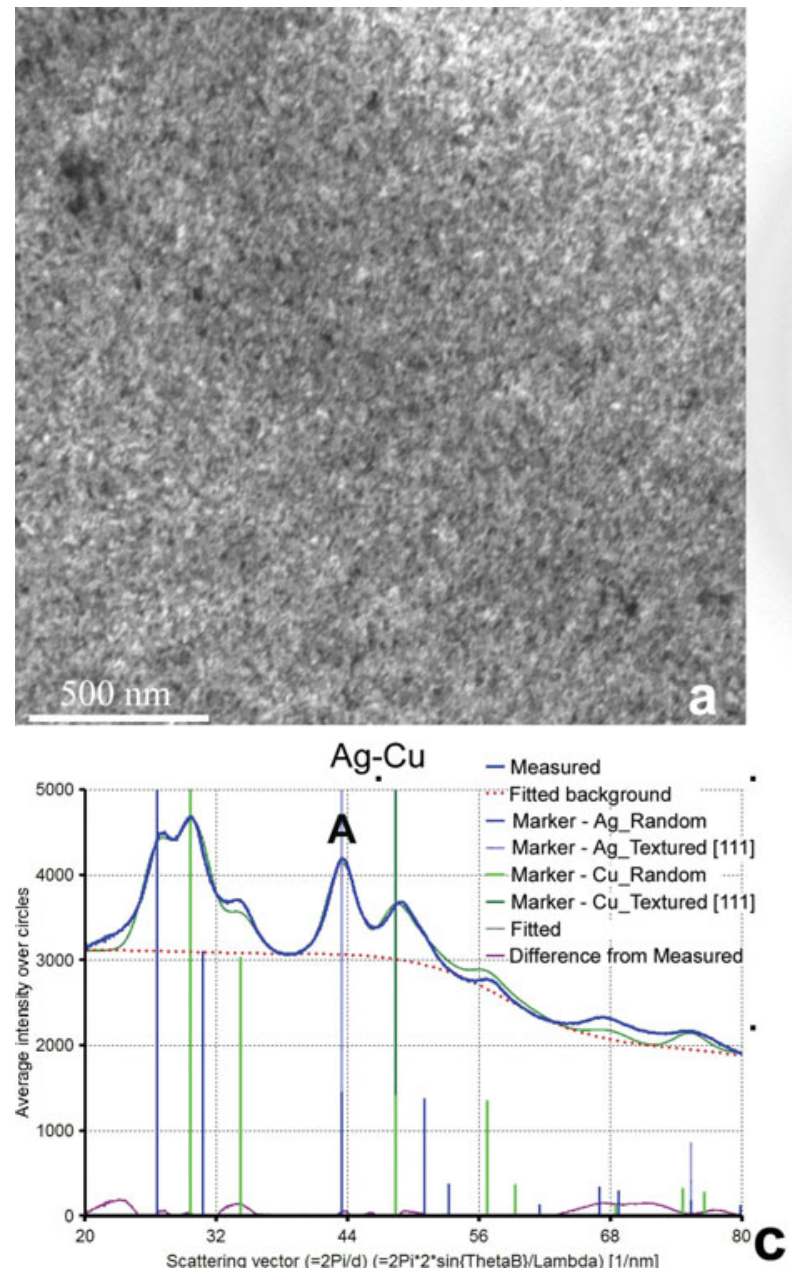

from EDS. The capability of the program to vary the lattice parameter of one of the phases is absolutely essential to a good fit. Without handling both the texture and the change in the lattice parameter, the fitting attempt would fail to converge to a visually acceptable agreement between fitted and measured distributions. The separated strong peak ("A" in Fig. 8c) cannot only be used to calibrate the camera length, but can also be used to calibrate peak width. That width can be "inherited" by the other peaks of the phase and also by other Markers during the next iterations of the fit (see the Serious Overlap; Manually Forced Starting Value for Peak Width; Inheritance section below).

\section{Effect of a Thin a-Carbon Support Layer}

In many TEM thin film samples, a thin supporting a-C layer is also present beneath the thin layer of interest. The scattering in this thin a-C layer modifies the shape of the background. That modification in shape is taken into account empirically in the present implementation, by fitting a cubic-Spline function to manually selected background points.

However, because both the a-C and the film of interest are thin, scattering in them must be kinematic and the effect of such a-C layer should be possible to remove by subtracting the diffracted distribution, measured from a

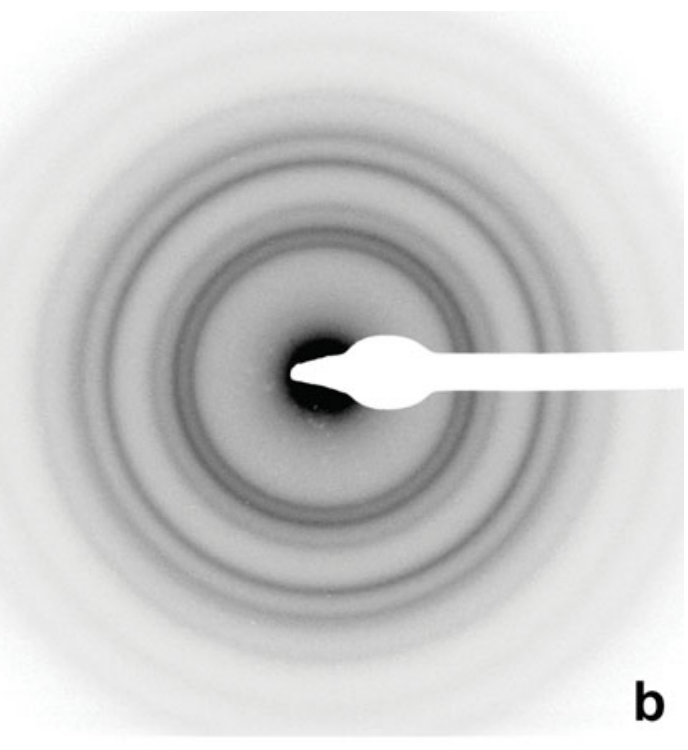

Figure 8. Ag- $\mathrm{Cu}$ nanocrystalline thin film. a: BF image of the layer. Grain size is $5 \mathrm{~nm}$. b: Measured SAED pattern. c: Processed distribution with fitted function.

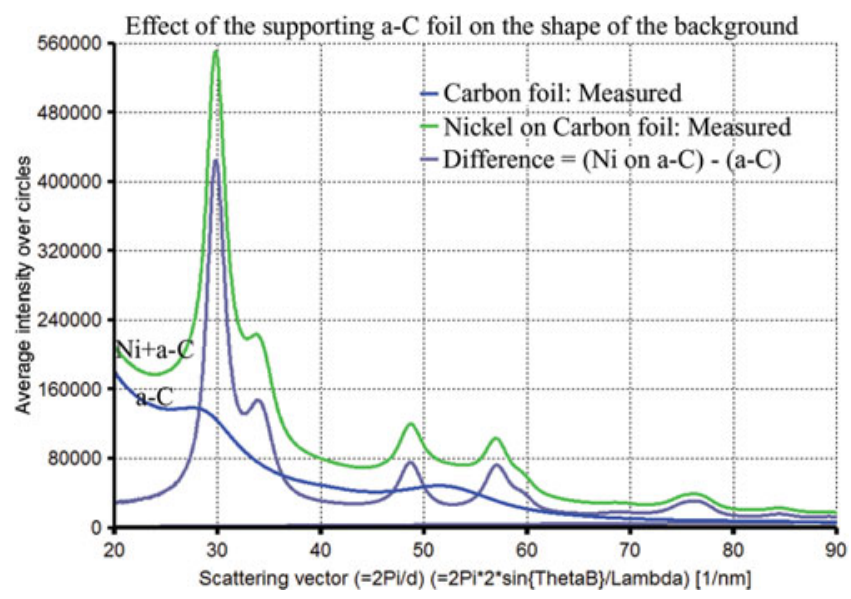

Figure 9. Effect of a thin a-C layer support on the shape and size of the background. The procedure of removing the effect of the thin supporting film is demonstrated here. The procedure is based on the assumption that scattering is kinematic in the thin layers, and consequently the effect of the components is additive. The three curves: SAED distribution from $10 \mathrm{~nm}$ Ni on $10 \mathrm{~nm}$ a-C; SAED distribution from a stand-alone a-C layer of the same thickness of $10 \mathrm{~nm}$ (prepared in the same batch as the one used as substrate for the Ni layer); difference of the previous two distributions, resulting in a good approximation to the SAED distribution of a self-supporting Ni layer of $10 \mathrm{~nm}$ thickness. 

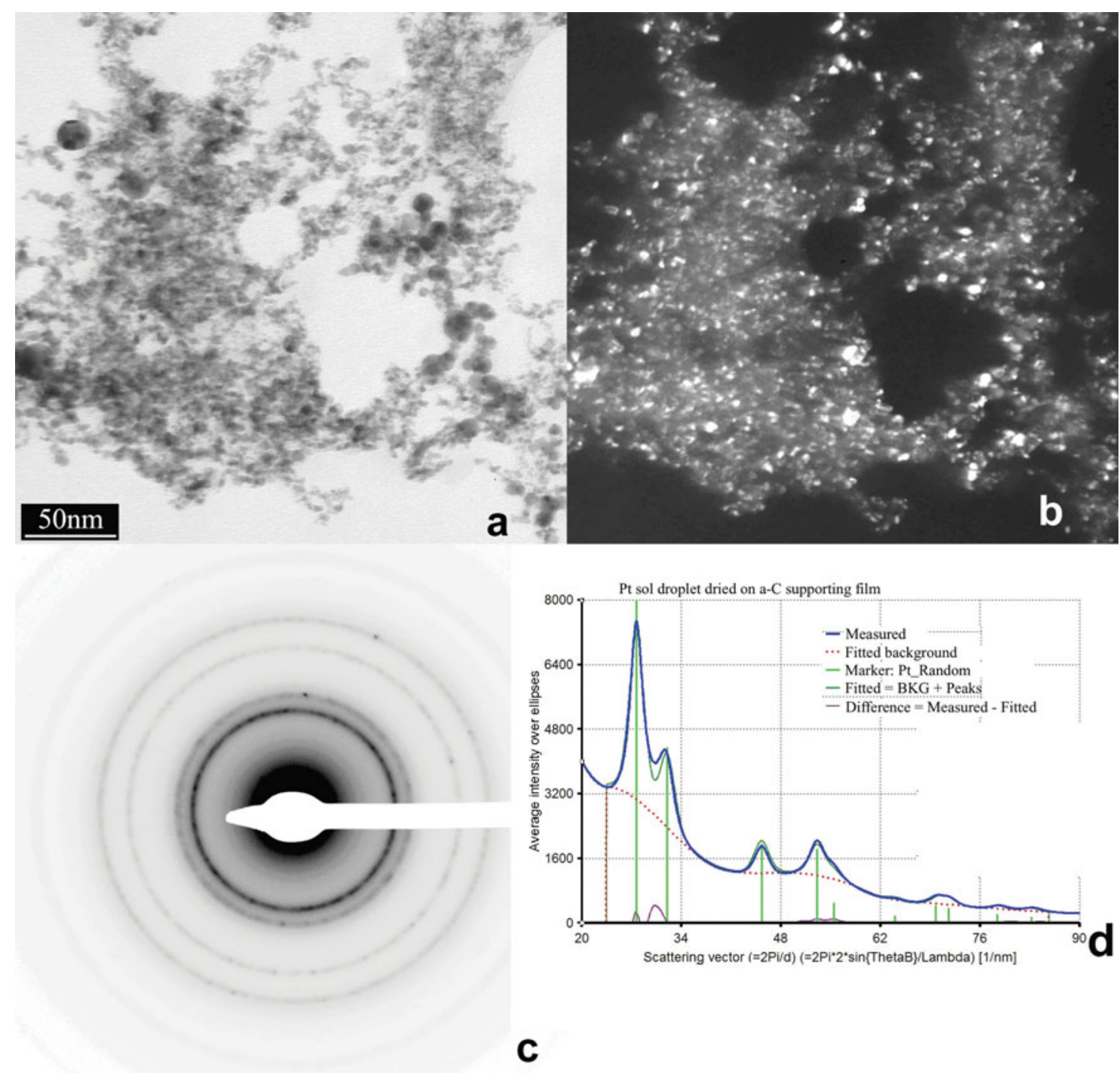

Figure 10. Dried droplet of Pt-sol on C-film. (The sample is produced by the Metal-Art Precious Metal Industrial Company, Hungary,. within the Metanano project.) Grain size is $3 \mathrm{~nm}(2.8 \pm 0.6 \mathrm{~nm})$. (a) BF, (b) DF, (c) SAED, and (d) fitted distribution, showing good agreement between measurement and kinematic calculation.

similar, stand-alone a-C layer under identical conditions. This effect was demonstrated by measuring two diffraction patterns, one from a $10 \mathrm{~nm}$ Ni layer on top of a $10 \mathrm{~nm}$ thick $\mathrm{a}-\mathrm{C}$ support and another one from an $\mathrm{a}-\mathrm{C}$ support layer of the same thickness (Fig. 9). The difference of these two patterns is a good approximation of the distribution of electron scattering from a self-supporting Ni layer with the same structure and thickness (Fig. 9). ${ }^{\mathrm{d}}$ The shape and size of the difference demonstrate that the kinematic approximation (i.e., the principle that the two contributions are additive) is not bad for these thin layers. For support with a known pattern, subtracting the patterns is the preferred method. However, the amorphous component may also be

\footnotetext{
${ }^{\mathrm{d}}$ Preparation of such a self-supporting Ni layer with identical structure and thickness may be difficult. For instance, if it is evaporated on $\mathrm{NaCl}$, both the structure and the grain size may differ (due to epitaxy, texture, etc.) from those obtained on a-C. Disintegration of the thin layer is also a problem when floating off the substrate.
}

present as an integral part of the sample (e.g., in the form of amorphous grain boundary phase). In such cases, empirical fitting with the Spline function only approximates the background effect of the amorphous part in the diffraction.

\section{Nanopowder with Random Orientation; Effect of the "Motif"}

The simplest type of sample was selected to test the agreement between kinematic calculation and measurement, namely a single-phase material with randomly oriented nanograins. The crystallographic structure is also simple, being cubic, with high symmetry and a consequent low number of lines. Two samples were examined. Both of them have fcc structures, but with different groups of atoms ("motif") at the lattice points. The first one is fcc-Pt, with one atom per lattice point. A droplet of Pt sol was dropped on a thin carbon support film and examined after the droplet had dried, leaving the nanoparticles in random 

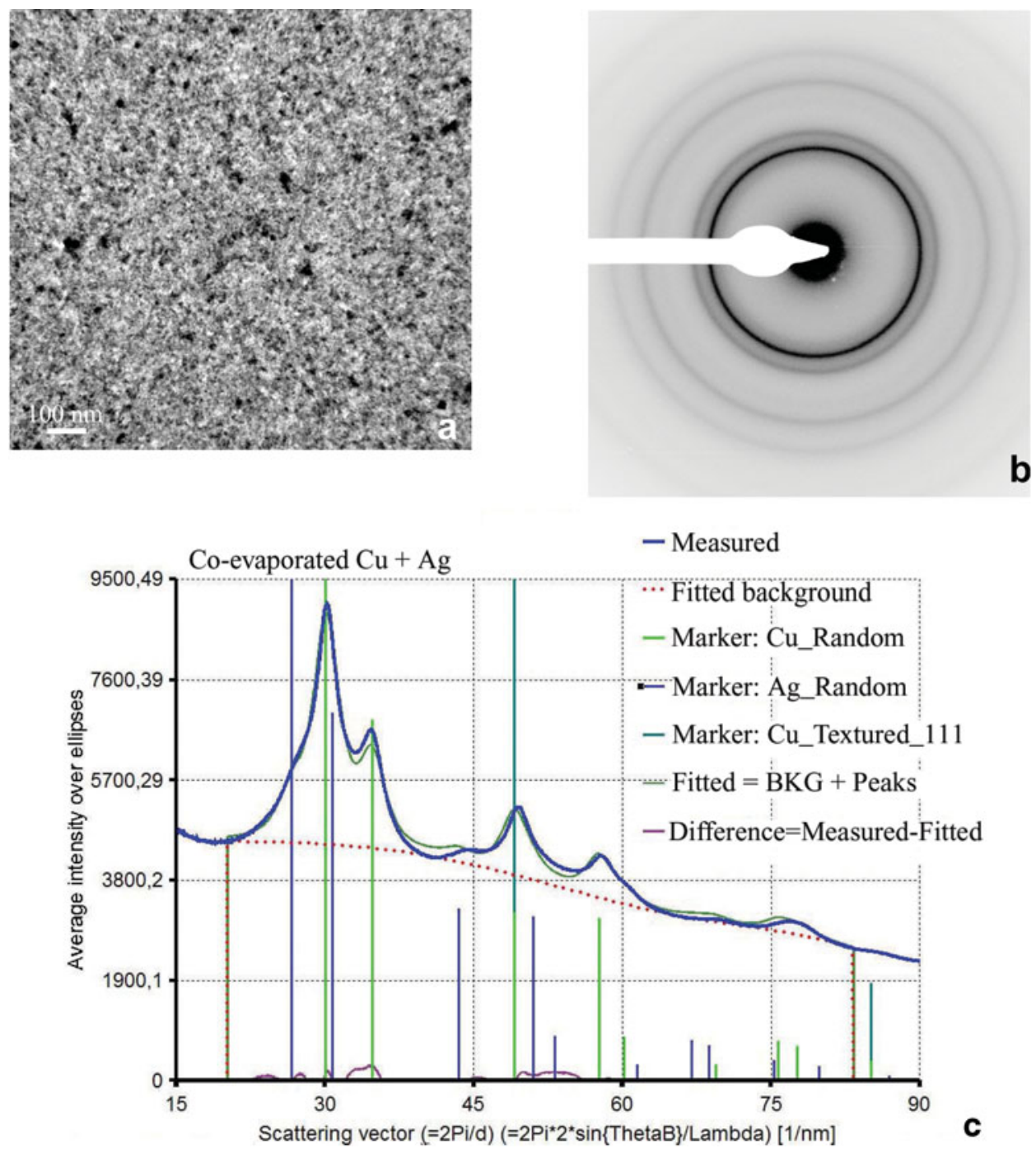

Figure 11. Texture analysis in nanocrystalline $\mathrm{Cu}-\mathrm{Ag}$ thin film. a: $\mathrm{BF}$ image of the layer, showing grain size of $2-5 \mathrm{~nm}$. b: Measured SAED pattern. c: Processed SAED pattern giving 4 vol\% Ag and showing that $42 \%$ of $\mathrm{Cu}$ is $\langle 111\rangle$ textured. Results are good agreement with both EDS and XRD.

distribution on the C-foil. Figure 10 shows grain size of $3 \mathrm{~nm}$ and uniform rings. Good fit in Figure 10d shows both that the orientation distribution is close to random, as expected, and that kinematic calculation approximates the experimentally observed values for such small grain size materials. The minor discrepancy between the positions of the (111) and (200) peaks may be an indication of small lattice distortion in the nanoparticles.

The other sample was a thin layer of $\mathrm{CrN}$ sputtered on a thin C-support film, as described in the Precision of Calibration: Absolute Measurement of Lattice Parameter section above. $\mathrm{CrN}$ also has an fcc structure, but two atoms belong to each lattice point. As a result, the line intensity ratios are very different, as seen in Figure 7 (compare to the single atom fcc Pt in Fig. 10). The good agreement between calculated and measured line intensity ratios in the figure again indicates both the randomness of the orientation distribution and the validity of the kinematic calculation for the small grain size of $10 \mathrm{~nm}$ in this sample.

\section{Texture in Nanocrystalline Thin Films}

The effect and handling of texture were already demonstrated in Figure 8. Another example of texture analysis is shown in Figure 11. Cu was co-evaporated with a small amount of Ag. EDS analysis resulted in $92 \mathrm{vol} \% \mathrm{Cu}$ and $8 \mathrm{vol} \%$ Ag. Processing the SAED pattern taken from the center of such a sample gave $96 \mathrm{vol} \% \mathrm{Cu}$ and $4 \mathrm{vol} \% \mathrm{Ag}$, in good agreement with the EDS result. The same analysis simultaneously gave that $42 \%$ of all $\mathrm{Cu}$ present is $\langle 111\rangle$ textured and the rest of the $\mathrm{Cu}(58 \%$ of it) is randomly oriented.

Another example for characterizing development of texture during growth in thin TiN film is examined in Figure 15, as presented in the Advantage of SAED over XRD section below.

\section{Two Phases, Medium Overlap}

A two-phase thin-film test sample was prepared by sequential evaporation of $\mathrm{Cr}$ and $\mathrm{Ag}$. EDS analysis gave $77 \mathrm{vol} \% \mathrm{Cr}$ 


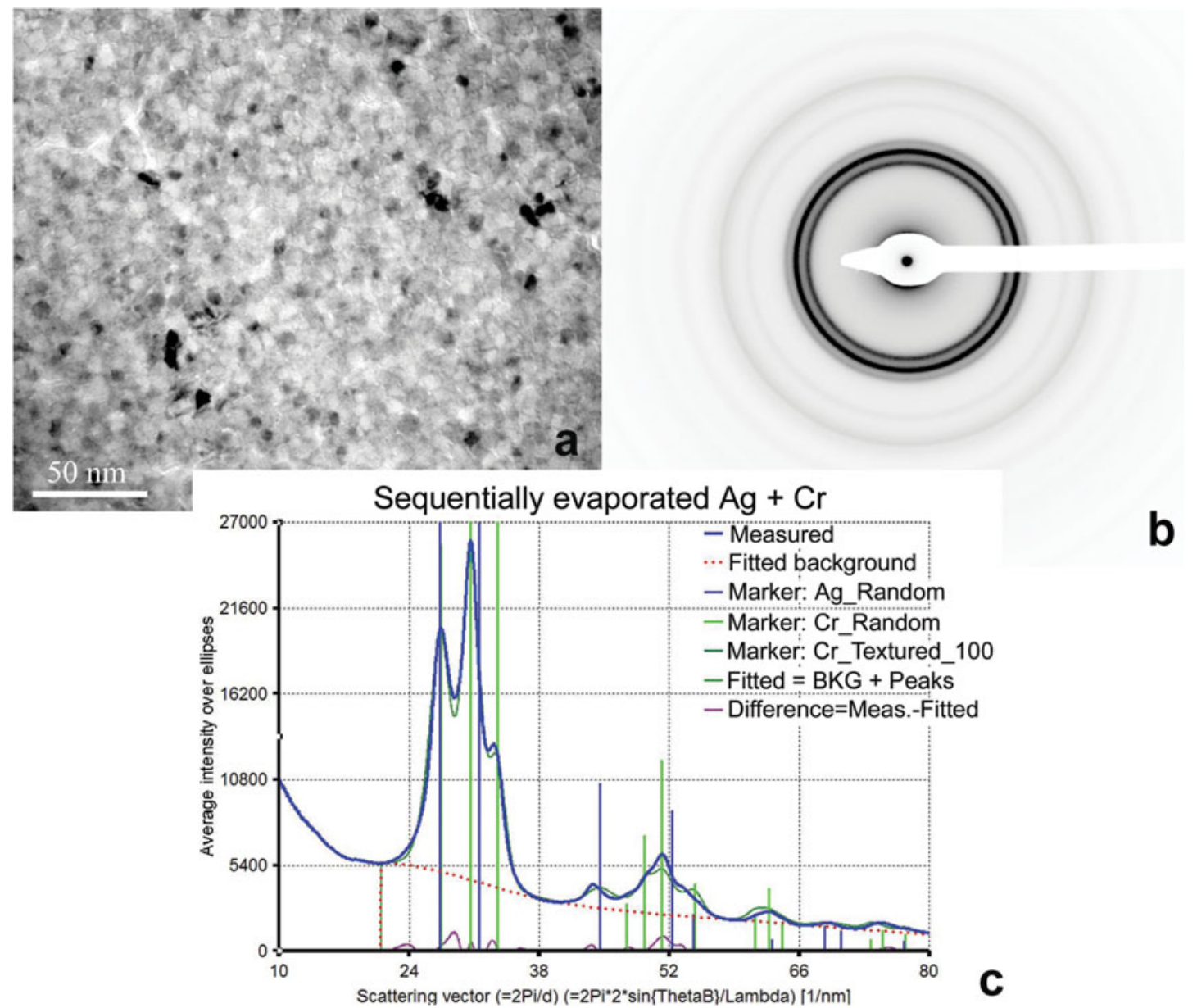

Figure 12. Example of evaluating textured patterns. a: BF image, showing a Cr-Ag thin film with grain size of 5-15 nm. b: Measured SAED pattern. c: Processed diffraction with fitted Model provided 70 vol\% Cr and 30 vol\% Ag. Agreement is acceptable with EDS composition analysis of $77 \mathrm{vol} \% \mathrm{Cr}$ and $23 \mathrm{vol} \% \mathrm{Ag}$.

and 23 vol\% Ag. The self-supporting thin film with grain size around 5-15 $\mathrm{nm}$ was analyzed by ProcessDiffraction (Fig. 12). Ag was randomly oriented, while $\mathrm{Cr}$ was significantly textured. The diffraction analysis provided $70 \mathrm{vol} \%$ $\mathrm{Cr}$ and $30 \mathrm{vol} \% \mathrm{Ag}$ (if texture was correctly taken into account), in agreement with EDS.

Another example of a two-phase sample is AlN-CrN (Fig. 13). A thin film sample is prepared by co-sputtering of $\mathrm{Cr}$ and $\mathrm{Al}$ in nitrogen atmosphere. The ratio of the metallic components is determined from EDS (75 at.\% Al and 25 at.\% Cr, both fully nitrided as shown by the diffraction pattern). The result is a two-phase, nanocrystalline sample with both fcc-CrN and h-AlN present. The lattice parameters of both phases changed due to mutual solution ( $\mathrm{Al}$ in $\mathrm{CrN}$ and $\mathrm{Cr}$ in $\mathrm{AlN}$ ).

A set of similar other test samples was also analyzed and results are shown in Table 2. Good agreement can be seen for the major components for all those studied systems. Estimates for both precision and detection limits are deduced below from Table 2. The results indicate that the phase fractions determined by our method are reliable (within 10-15\% relative) for major components in case of medium overlap.

\section{Serious Overlap; Manually Forced Starting Value for Peak Width; Inheritance}

When two phases with identical structure and only slightly different lattice parameter are simultaneously present, most of the rings in the $2 \mathrm{D}$ pattern are not well separated, so the

Table 2. Examples of Analyzed Two-Phase Test Layers.

\begin{tabular}{llcc}
\hline Sample \# & $\begin{array}{c}\text { Vol\% } \\
\text { (Quartz/EDS) } \\
( \pm 1 \sigma)\end{array}$ & $\begin{array}{c}\text { Vol\% } \\
(\text { SAED }) \\
( \pm 1 \sigma)\end{array}$ \\
\hline 1 & Phase & $50 \pm 5$ & $49 \pm 5$ \\
& Al (fcc) & $50 \pm 5$ & $51 \pm 5$ \\
2 & NiO (fcc) & $77 \pm 3$ & $73 \pm 3$ \\
3 & Cr (cP8) & $23 \pm 3$ & $27 \pm 3$ \\
4 & Ag (fcc) & $2 \pm 2$ & 0 \\
4 & Cr (cP8) & $98 \pm 2$ & 100 \\
& Ag (fcc) & $76 \pm 5$ & $83 \pm 5$ \\
& Ni (fcc) & $24 \pm 5$ & $17 \pm 5$ \\
& Ag (fcc) & $95 \pm 4$ & $96 \pm 4$ \\
& Ni (fcc) & $5 \pm 4$ & $4 \pm 4$ \\
\hline
\end{tabular}



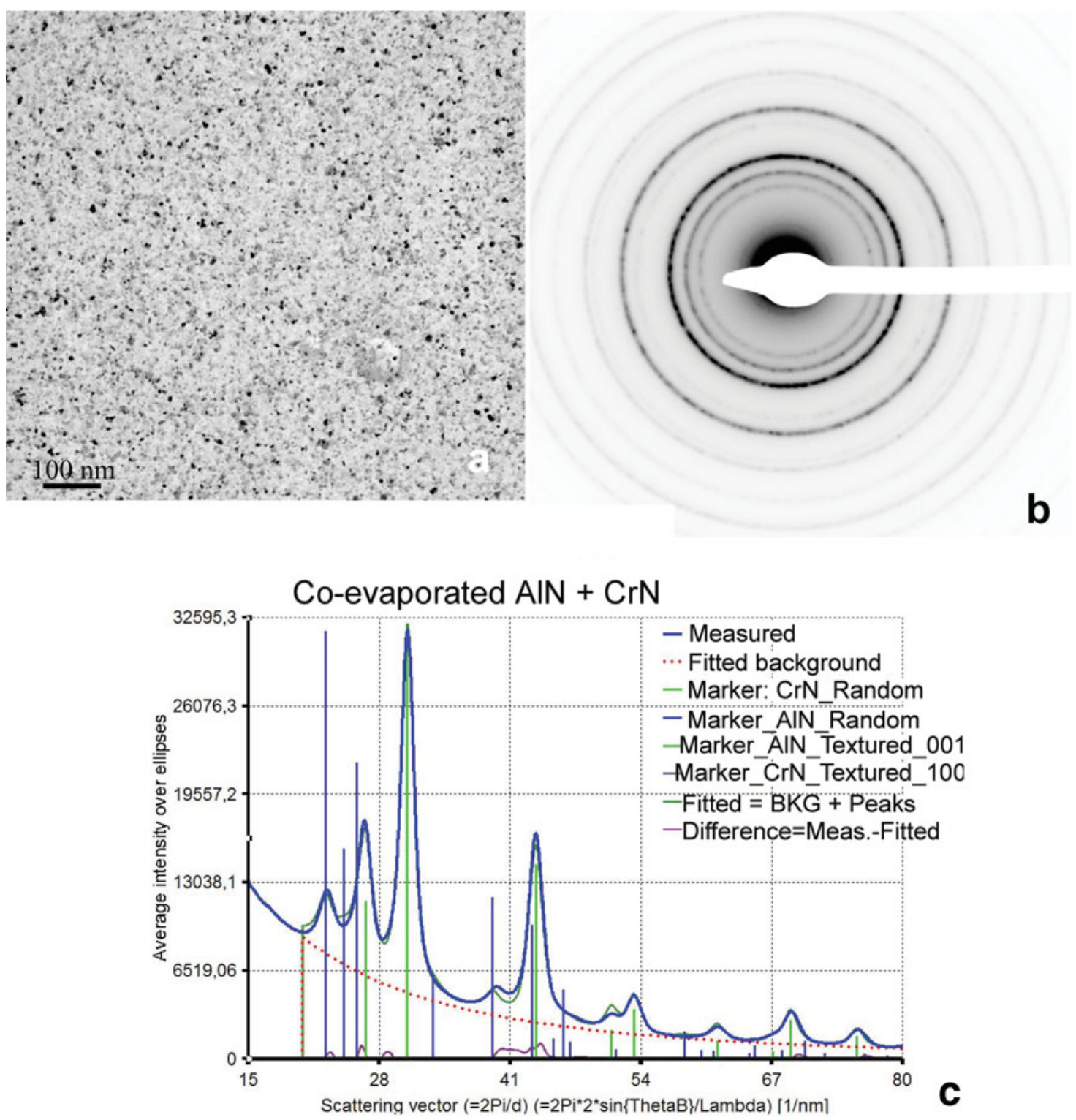

Figure 13. A thin film sample prepared by co-sputtering of $\mathrm{Cr}$ and $\mathrm{Al}$ in nitrogen atmosphere. The ratio of the metallic components is determined from $\operatorname{EDS}(75$ at.\% $\mathrm{Al}$ and 25 at.\% $\mathrm{Cr}$, both fully nitrided as shown by the diffraction pattern). The result is a two-phase, nanocrystalline sample with both fcc-CrN and h-AlN present. The lattice parameters of both phases changed due to mutual solution ( $\mathrm{Al}$ in $\mathrm{CrN}$ and $\mathrm{Cr}$ in $\mathrm{AlN}$ ). a: BF image, showing a grain size of $10 \mathrm{~nm}$. b: Measured SAED pattern. c: Processed distribution, with fitted Model functions.

peaks on the $1 \mathrm{D}$ distribution overlap. In such cases the automatic fitting procedure might fall into a local minimum, and only manual intervention can help the fitting process to find the global minimum. As you can see below, the principles of that manual intervention are clear, and by intervening correctly the most appropriate solution of the phase analysis problem can be found. Correctness of the solution is always checked visually by the quality of the fit. Analysis of a Ni/Cu multilayer is presented as an example here. The nominal composition was measured by EDS analysis. The lattice parameter of fcc copper is $3.604 \AA$, while that of fcc Ni is $3.529 \AA$. The degree of overlap is demonstrated in Figure 14a. If fitting is attempted with all parameters released for variation, the result is unacceptable. Practically one phase is detected with increased peak width that provides a minimized goodness-of-fit parameter, but physically meaningless, which is also reflected in the shape of the fitted distribution (Fig. 14a). In such problematic cases, the peak width and peak shape must be determined first from a narrow interval, where the overlap is negligible. That value of peak width (and peak shape) must not be changed during later fitting to a wider interval that contains all the important peaks. That step of fitting is illustrated in Figure $14 \mathrm{~b}$. To avoid singular matrix, the fitting to the narrow interval of a single, separated peak (Fig. 14b) is done with only one Marker active. Selecting a narrow interval mounts to "forcing the peak width and peak shape manually." After extending the fitting interval to all interesting peaks, the 
a

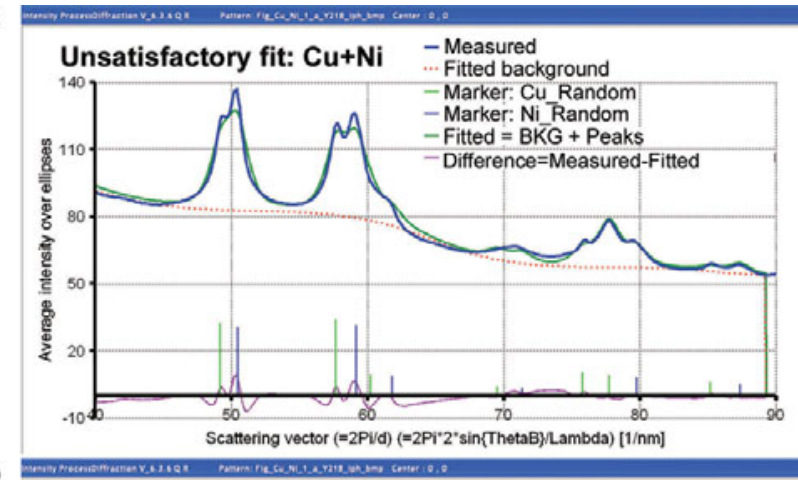

b

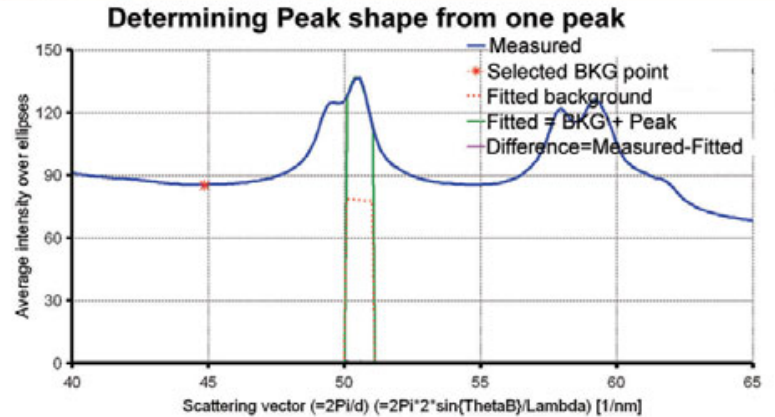

c

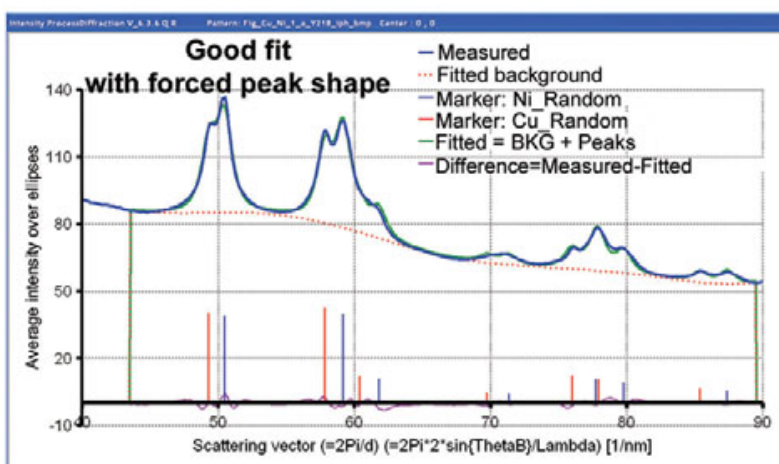

Figure 14. Example of overcoming a serious overlap by using inherited peak shapes. $\mathrm{Cu}-\mathrm{Ni}$ multilayer sample was analyzed with EDS, resulting in 50 at.\% Cu-50 at.\% Ni, which corresponds to 52 vol\% $\mathrm{Cu}-48$ vol\% Ni. Illuminated area for EDS corresponded to the area selected by SAA for diffraction analysis. Unconstrained fit resulted in bad peak shapes, so result was disregarded. Peak shape (including peak width) was fixed to match a "separated" peak in panel $\mathbf{b}$ (line $\{220\}$ of $\mathrm{Ni}$ ) and that shape was forced on all other peaks in the distribution (the other peaks "inherited" this shape). Good fit with that inherited peak shapes is shown in panel c, and it resulted in $58 \mathrm{vol} \% \mathrm{Ni}$ and $42 \mathrm{vol} \% \mathrm{Cu}$. The rather large difference between the elemental composition by EDS and the phase composition by SAED might (partially) be attributed to the fact that the FIB-cut sample was mounted on $\mathrm{Cu}$ grid, so stray radiation may have caused slight false increase in $\mathrm{Cu}$ content measured by EDS, but this assumed effect could not be taken into account.

same peak shape and peak width are used as default values for all the other peaks of the Marker. Inheritance means that the subsequently defined Markers can be forced to "inherit" the same parameters. The improved fit is shown in Figure $14 \mathrm{c}$. The result of the analysis is $42 \mathrm{vol} \% \mathrm{Cu}$. The rather large difference between the elemental composition by EDS $(52 \mathrm{vol} \% \mathrm{Cu})$ and the phase composition by SAED might
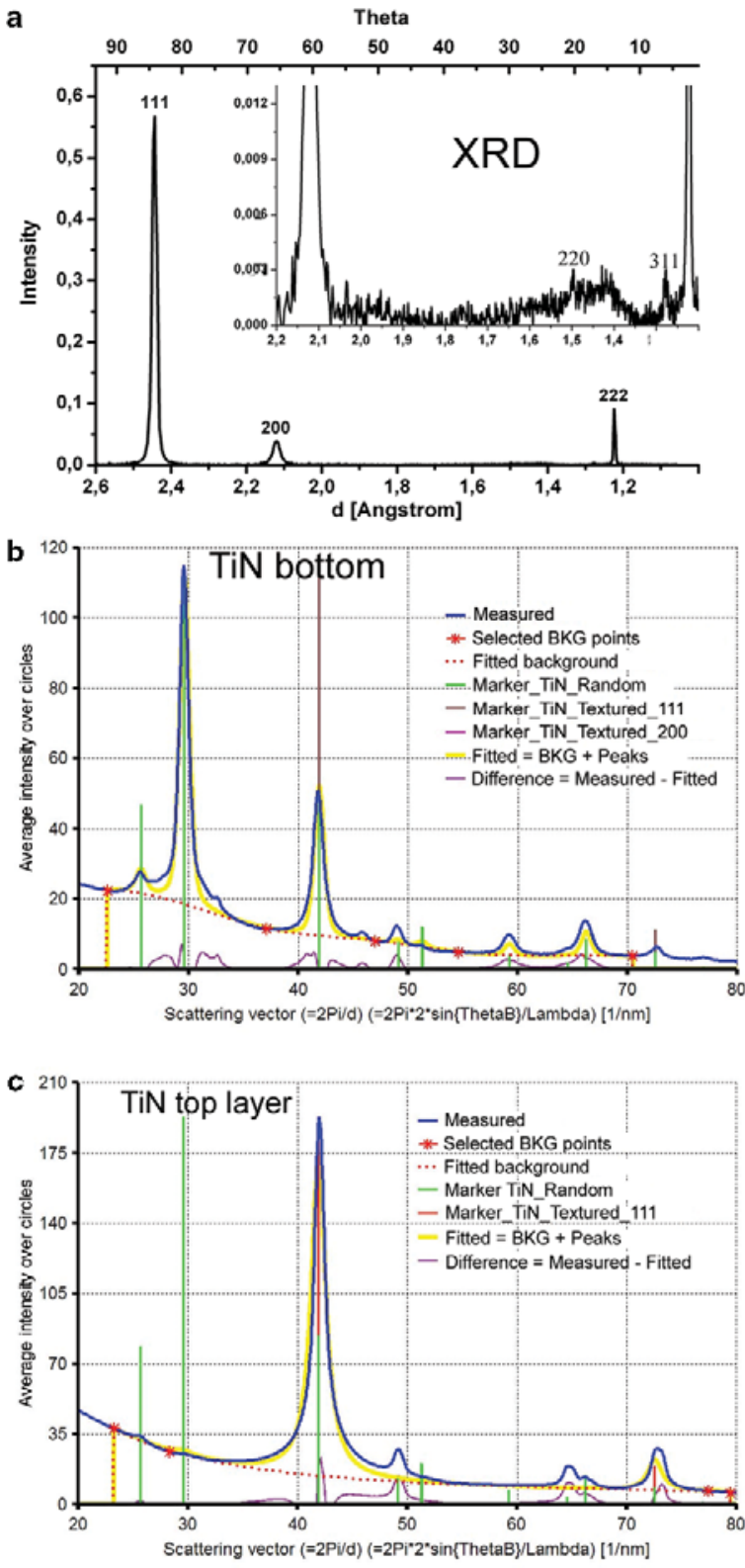

Figure 15. Illustrates the advantage of SAED in the TEM over XRD. a: XRD of a $1 \mu \mathrm{m}$ thick TiN layer (on a Si substrate). The globally significant $\langle 111\rangle$ and $\langle 200\rangle$ texture components are only seen. The inset shows that other peaks are just above the detection level under the given experimental conditions. b: SAED intensity distribution taken from a lateral section at the bottom $100 \mathrm{~nm}$ part of the layer. Analysis shows that $13 \mathrm{vol} \%$ of the TiN in that section is made of random randomly oriented nanograins, 81 vol $\%$ is 200 -textured, while 6 vol $\%$ is 111 -textured. c: SAED intensity distribution taken from a lateral section at the top $100 \mathrm{~nm}$ part of the layer. Analysis shows that $100 \%$ is 111 textured.

(partially) be attributed to the fact that the focused ion beam (FIB)-cut sample was mounted on a $\mathrm{Cu}$ grid. Stray radiation may have caused an increase in $\mathrm{Cu}$ content as measured by EDS. Alternatively the difference can be interpreted as an indication that relative accuracy is not better than $20 \%$ in this case. 


\section{Advantage of SAED over XRD}

There are two main advantages of electron diffraction in a TEM over XRD. First, much smaller volumes can be studied. Thin layers of a few tens of nanometer thickness can be studied individually and separately in the TEM enabling precise and documented identification of the inspected volume. This is done by preparing thin lateral sections from different depths of a thick layer if needed. A cross-sectional TEM sample is compared to these lateral sections to give a complete characterization. Since the entire excited volume is within these very thin lateral layers, small fractions of these tiny volumes are seen in the SAED patterns. That small fraction can either be another phase or a texture component that amounts to a low volume fraction of the entire volume that is around the detection level of XRD. This resolution easily facilitates following up phenomena of structure formation in such details, which is impossible with usual XRD. Second, the volume to be examined can be selected in a TEM image of the structure.

An example of the above-mentioned advantage is demonstrated in Figure 15. A TiN sample was prepared with reactive sputtering of titanium in nitrogen atmosphere on a Si substrate. It is taken from a systematic series of samples used to understand texture development as a function of experimental parameters, as elaborated in Ehiasarian et al. (2011). The data in our Figure 15 here provide complementary material to Figures 11 and 12 and Table II of the cited article. The entire layer thickness is $1 \mu \mathrm{m}$. XRD of the layer shows strong $\langle 111\rangle$ texture with minor $\langle 200\rangle$ texture component (Fig. 15a). ${ }^{\text {e }}$ Cross-section TEM image (Fig. 11 of Ehiasarian et al., 2011) shows an inhomogeneous structure that is in agreement with the structure development models. The bottom layer of about $50-100 \mathrm{~nm}$ thickness is small grained, which turns into V-shaped columns, characteristic of competing growth in zone-T of the structure-zone model (Barna \& Adamik, 1998; Petrov et al., 2003). Lateral sections were cut from the layer at the bottom, at the middle, and at the top of the ( $1 \mu \mathrm{m}$ thick) layer. Their structures (grain sizes) are shown in Figure 12 of Ehiasarian et al. (2011) demonstrating that the lateral grain size continuously grows from $\sim 3 \mathrm{~nm}$ at the bottom to $100 \mathrm{~nm}$ at the top. Figures $15 \mathrm{~b}$ and $15 \mathrm{c}$ present the processed electron diffraction intensity distributions from the bottom and from the top lateral sections, respectively. The thin bottom layer in Figure $15 \mathrm{~b}$ is dominated by the $\langle 200\rangle$ texture, with an additional small fraction of randomly oriented grains. Those grains with orientation different from the $\langle 200\rangle$ texture

\footnotetext{
${ }^{\mathrm{e}}$ The reader is reminded that not the same planes reflect in XRD and in the TEM if identically oriented lateral layers are examined. XRD sees the layers parallel to the substrate surface. For the special case of cubic crystals (where directions normal to a plane have the same directional indices as the Miller indices of the plane), a single peak in the XRD corresponds to the texture axis. This is completely different for SAED in the TEM. The Bragg angles extend to a few milliradians, so the planes in reflecting conditions are almost parallel to the electron beam (i.e., normal to the substrate). Consequently, no peak with the indices of the texture axis can be observed in SAED.
}

provide the seed of the V-shaped grains that later overgrow the $\langle 200\rangle$ grains and rapidly turn the texture into $\langle 111\rangle$ that dominates the bulk of the volume for the entire layer. The top layer in Figure 15c shows perfect $\langle 111\rangle$ texture, representing the end product of structure development in agreement with XRD.

We can draw several conclusions from those observations. First, the main components of the structure detected by XRD are in agreement with the TEM observations and SAED. Second, the XRD data do not give information about the localization of the $\langle 111\rangle$ and $\langle 200\rangle$ components that is important for the interpretation of the phenomena of structure formation. Third, XRD could hardly detect the tiny fraction of grains with orientations different from the two main texture components. ${ }^{f}$ The inset in Figure 15a shows that both 220 and 311 peaks are also present at the XRD, but their intensities are just above the background noise. ${ }^{\mathrm{g}}$ The presence of these additional peaks is not only shown in the SAED pattern from the lateral section at the TEM, but their existence also provides the clue to understand structure formation. They provide the seeds for the later $\mathrm{V}$-shaped crystals that swap the structure from the initial mainly $\langle 200\rangle$ texture into $\langle 111\rangle$ texture. The advantage of SAED over $\mathrm{XRD}$ is obvious from the example.

\section{Detection Limits}

The exact value of the detection limit must obviously be a function of both the material and the structure of the phases present. However, the results give an estimate for the minimum volume fraction that can be detected (calculated for the crystalline components only in the nanocrystalline sample). When a weakly scattering phase is only present by a few percent within a more strongly scattering matrix, like $2 \% \mathrm{Cr}$ in Ag (Table 2), we are unable to detect it because no statistically significant peak is seen for the $\mathrm{Cr}$ phase in the measured distribution. However, when the amount of a more strongly scattering phase reaches $5 \%$ in a less strongly scattering matrix (such as $5 \% \mathrm{Ag}$ in $\mathrm{Ni}$ in Table 2), it is possible to quantify it. As a rule of thumb (deduced from a limited number of experiments), we estimate the detection limit to be 5 vol\%.

\section{Precision and Accuracy}

Precision of the processing method is estimated from comparing results of repeated fits to the same distribution. With identical fitting parameters the method converges to the

\footnotetext{
${ }^{\mathrm{f}}$ Since $\cong 1 / 10$ th of the entire layer contains the random component and the relative intensity of the next reflection to be detected (i.e., 220) is $45 \%$, while that of the 200 is $100 \%$, the 220 peak should be one and a half orders of magnitude less intense than the observed (small) 200 peak in Figure 15a and that is just at the detection limit of XRD with the given experimental conditions.

g The net intensities of the peaks from the XRD are $100 \%$ for 111 reflection, $6.9 \%$ for $200,0.25 \%$ for 220 , and $0.4 \%$ for 311 . That roughly corresponds to $95 \mathrm{vol} \%$ of 111 textured, 4 vol\% of 200 textured, and $1 \mathrm{vol} \%$ of randomly oriented component, in agreement with our estimate from electron diffraction.
} 
same result (if started from the same starting condition). However, when started from different estimates of parameters and the parameters to optimize are selected differently, we may end in different local minima. It is the responsibility of the operator to decide whether the fit is good enough (by visual observation) and also whether the resulting parameters (e.g., thermal parameters, grain size parameters, etc.) look meaningful.

Accuracy is evaluated by comparing the result of ProcessDiffraction to the nominal phase composition values that were determined by thickness monitor and EDS.

That empirical estimate shows a relative accuracy of $10-15 \%$ for major components. Thus, for a nominal composition of $50 \mathrm{vol} \%$, we expect our method to yield a result in the range $43-57 \mathrm{vol} \%$. Error propagation in our method plays a very similar role to that in the Cliff-Lorimer method for EDS analysis of thin films in the TEM. Since the result is normalized to $100 \%$ in both methods, the size of the error is mainly determined by the minor component if the fraction of component " $\mathrm{A}$ " is much smaller than that of component " $\mathrm{B}$ " in a two-component sample. For example, if the true phase composition is $95 \%$ " $\mathrm{A}$ " and $5 \%$ " $\mathrm{B}$," then a $10 \%$ relative error in the measured value of " $\mathrm{A}$ " would only induce $\pm 0.5 \%$ variation in absolute concentrations, and the result would scatter between $94.5 \%$ "A" $+5.5 \%$ "B" and 95.5\% "A" $+4.5 \%$ "B" due to the normalization (provided the measured value for "B" did not change simultaneously). The minor components are generally measured with a reduced precision. If the measured value for " $\mathrm{B}$ " is in $80 \%$ relative error that would induce $\pm 4 \%$ variation in absolute concentrations and the result would scatter between $91.3 \%$ "A" + 8.7\% "B" and 99\% "A" + 1\% "B" due to the normalization. That effect was taken into account in assigning error limits to the values in Tables 1 and 2.

\section{Conclusions}

A method to determine phase fractions and special texture components from selected area electron diffraction patterns of nanocrystalline thin samples was presented in a series of three articles. Different aspects of applying this method were demonstrated here in part III. The method can be applied to a wide range of simple structures. The structures examined required at most four model functions (Markers), e.g., two phases, both of them showing partial texture. The examined crystal structures were cubic and hexagonal. In principle, phase fractions of any crystal systems should be possible to determine. However, it must be recognized that due to the limited spectral (= angular) resolution of electron diffraction from nanocrystalline samples, a diffraction pattern with too many lines should pose too many uncertainties due to the numerous peak overlaps. That is why low symmetry phases could not be evaluated with similar success.

Grain size (in beam direction) is currently determined from the Blackman correction. Examining changes in peak widths due to grain size and crystal defects is planned for later implementations. The applicable range of grain size in beam direction is extended to $10-30 \mathrm{~nm}$ by incorporating the Blackman correction for dynamic effects. However, it is emphasized that our "grain size in beam direction" is a quantity that is difficult to correlate with the usual grain size or sample thickness. In the case of spherical grains, it gives the usual grain size. In the case of columnar grains, it depends on the direction-how the thin TEM sample was cut from the bigger sample. In case of a cross section, parallel to the columns, it is the column width. In case of a lateral section, perpendicular to the columns, it is the sample thickness. In the case of an oblique section, our "grain size in beam direction" does not correspond to either of them.

Determination of thermal parameters is part of the method. However, the values of these parameters must be handled with caution, due to the interaction of parameters during fitting.

For samples that contain a small number of high symmetry phases, the relative accuracy is $10-15 \%$ for major components. The detection limit is about 5 vol\% for significantly scattering phases and is undetermined for weakly scattering ones.

The method can successfully determine several difficult parameters simultaneously. Changes in lattice parameter can be handled with simultaneous determination of phase fractions and fractions of axially textured components.

\section{ACKNOWLEDGMENTS}

Z.C. and F.M. acknowledge the Bolyai Scholarship of the Hungarian Academy of Sciences.

\section{REFERENCES}

BARNA, P.B. \& ADAMIK, M. (1998). Fundamental structure forming phenomena of polycrystalline films and the structure zone models. Thin Solid Films 317, 27-33.

Ehiasarian, A.P., Vetushia, A., Aranda Gonzalvo, Y., Sáfrán, G., SzéKely, L. \& BARnA, P.B. (2011). Influence of high power impulse magnetron sputtering plasma ionization on the microstructure of TiN thin films. J Appl Phys 109, 104314.

Goldstein, A.N., Echer, C.M. \& Alivisatos, A.P. (1992). Melting in semiconducting nanoncrystals. Science 256, 1425-1427.

LÁBÁR, J.L. (2008). Electron diffraction based analysis of phase fractions and texture in nanocrystalline thin films, Part I: Principles. Microsc Microanal 14, 287-295.

LÁbÁR, J.L. (2009). Electron diffraction based analysis of phase fractions and texture in nanocrystalline thin films, Part II: Implementation. Microsc Microanal 15, 20-29.

Petrov, I., Barna, P.B., Hultman, L. \& Greene, J.E. (2003). Microstructural evolution during film growth. J Vac Sci Technol A 21, S117-S128.

Weickenmeier, A. \& KoHL, H. (1991). Computation of absorptive form factors for high-energy elecron diffraction. Acta Crystal$\log r$ A 47, 590-597.

Williams, D.B. \& Carter, C.B. (1996). Transmission Electron Microscopy; A Textbook for Materials Science, p. 285. New York, London: Plenum Press. 